\title{
Impact of Pharmacist Involvement in the Transitional Care of High-Risk Patients Through Medication Reconciliation, Medication Education, and Postdischarge Call-Backs (IPITCH Study)
}

\author{
Arti Phatak, PharmD, BCPS ${ }^{1}$, Rachael Prusi, PharmD¹, Brooke Ward, PharmD, BCPS ${ }^{1}$, Luke O. Hansen, MD, MPH², \\ Mark V. Williams, $\mathrm{MD}^{3}$, Elizabeth Vetter, PharmD¹, Noelle Chapman, PharmD1, Michael Postelnick, RPh, BCPS AQ ID ${ }^{1 *}$
}

\begin{abstract}
${ }^{1}$ Pharmacy Department, Northwestern Memorial Hospital, Chicago, Illinois; '2Division of Hospital Medicine, Department of Medicine, Northwestern University Feinberg School of Medicine, Chicago, Illinois; ' ${ }^{3}$ Department of Internal Medicine, University of Kentucky, Lexington, Kentucky.
\end{abstract}

BACKGROUND: Previous data suggest that direct pharmacist interaction with patients through medication reconciliation, discharge counseling, and postdischarge phone calls decreases the number of adverse drug events (ADEs) and plays an overall positive role in transitional care. Previous studies have evaluated pharmacist involvement in improving transitional care, but these studies did not include multiple postdischarge follow-up phone calls.

OBJECTIVES: The objectives of this study were to assess the impact of pharmacist involvement in transitions of care as measured by decreased medication errors (MEs) and ADEs, patients' knowledge related to communication about their medications as measured by improvement in the Hospital Consumer Assessment of Healthcare Providers and Systems (HCAHPS) scores, and 30-day all-cause inpatient readmissions and emergency department (ED) visits.

METHODS: This was a prospective, randomized, singleperiod longitudinal study that occurred from November 2012 through June 2013 at an urban, tertiary, academic medical center. Patients admitted to 2 designated internal medicine units on high-risk medications or with greater than 3 prescription medications upon discharge were included for randomization. The control group received the usual hospital standard of care. The study group received face- to-face medication reconciliation, a patient-specific pharmaceutical care plan, discharge counseling, and postdischarge phone calls on days 3,14 , and 30 to provide education and assess study endpoints.

RESULTS: A total of 278 patients were included in the final analysis, with 141 in the control group and 137 in the study group. Fifty-five patients (39\%) in the control arm experienced an inpatient readmission or ED visit within 30-days postdischarge compared to 34 patients $(24.8 \%)$ in the study arm $(P=0.01)$. Eighteen patients $(12.8 \%)$ in the control group experienced an ADEs or MEs compared to 11 patients $(8 \%)$ in the study group $(P>0.05)$. The HCAHPS scores during the study period showed a $9 \%$ improvement for the assessed questionnaire domain $(P>0.05)$.

CONCLUSIONS: This study demonstrated that pharmacist involvement in hospital discharge transitions of care had a positive impact on decreasing composite inpatient readmissions and ED visits. Statistically significant difference in medication-related events and HCAHPS scores were not observed. Patients with moderately complex medication regimens benefited from a continuity of care involving a pharmacy team during transitions in care. Journal of Hospital Medicine 2016;11:39-44. (C) 2015 Society of Hospital Medicine
Hospital readmissions have a significant impact on the healthcare system. Medicare data suggest a $19 \%$ allcause 30 -day readmission rate, of which $47 \%$ may be preventable. ${ }^{1,2}$ The Centers for Medicare \& Medicaid Services continue to expand their criteria of disease states that will be penalized for readmissions, now reducing hospital reimbursement rates up to $3 \%$.

*Address for correspondence and reprint requests: Michael Postelnick, RPh, BCPS AQ ID, Clinical Practice Manager, Senior Infectious Diseases Pharmacist, Northwestern Memorial Hospital, 251 E. Huron LC-700, Chicago IL 60014; Telephone: 312-926-7965; Fax: 312-926-7956; E-mail:mpostel@nm.or

Additional Supporting Information may be found in the online version of this article

Received: April 15, 2015; Revised: September 1, 2015; Accepted: September 9, 2015

2015 Society of Hospital Medicine DOI 10.1002/jhm.2493

Published online in Wiley Online Library (Wileyonlinelibrary.com).
Pharmacists, by optimizing patient utilization of medications, can play a valuable role in contributing to preventing readmissions. ${ }^{3}$

Lack of acceptable transitional care is a serious problem that is consistently identified in the literature. ${ }^{4}$ Transitional care involves 3 domains of transfer: information, education, and destination. A breakdown in any of these components can negatively impact patients and their caregivers.

Prior studies consistently demonstrated a high likelihood of adverse drug events (ADEs) and patients' lack of knowledge regarding medications postdischarge, both of which can lead to readmission. Forster and colleagues found that $19 \%$ to $23 \%$ of patients experienced an ADE within 5 weeks of discharge from an inpatient visit, $66 \%$ to $72 \%$ of which were drug related, and approximately one-third were deemed preventable. 5,6 One survey found that less than $60 \%$ 
of patients knew the indication for a new medication prescribed at discharge, whereas only $12 \%$ reported knowledge of an anticipated ADE. ${ }^{7}$

Pharmacists can play a large role in the information and education aspect of transitional care. Previous studies demonstrate that pharmacist involvement in the discharge process can reduce the incidence of ADEs and have a positive impact on patient satisfaction. There are conflicting data regarding the effect of comprehensive medication education and follow-up calls by pharmacy team members on ADEs and medication errors (MEs). ${ }^{3,8,9}$ Although overall pharmacist participation has shown positive patient-related outcomes, the impact of pharmacists' involvement on readmissions has not been consistently demonstrated. ${ }^{10-14}$

Our study evaluated the impact of the pharmacy team in the transitions-of-care settings in a unique combination utilizing the pharmacist during medication reconciliation, discharge, and with 3 follow-up phone call interactions postdischarge. Our study was designed to evaluate the impact of intensive pharmacist involvement during the acute care admission as well as for a 30-day time period postdischarge on both ADEs and readmissions.

\section{METHODS}

All patients were admitted to hospitalist-based internal medicine units at Northwestern Memorial Hospital, an 894-bed academic medical center located in Chicago, Illinois. Patients were randomized by study investigators using a random number generator to either the usual care or intervention arms and then evaluated each day for eligibility to participate in the study. Patients remained blinded throughout the study. Patients met inclusion criteria if they were discharged to home and either discharged on greater than 3 scheduled prescription medications or discharged with at least 1 high-risk medication. Highrisk medications were classified as anticoagulants, antiplatelets (eg, aspirin and clopidogrel), hypoglycemic agents (eg, insulin), immunosuppressants, or antiinfectives. Patients also needed to participate in a minimum of 1 postdischarge phone call or experience an emergency department (ED) visit or readmission within 30 days of discharge to meet inclusion criteria. Exclusion criteria included: impaired cognition based on Mini-Cog screening assessment scale, unable or unwilling to provide informed consent, lack of a personal phone number, non-English speaking, subsequent elective readmission within 30 days of initial visit, more than 3 previous hospital admissions in the past 2 months, palliative care or home/skilled nursing hospice, anticipated length of survival less than 3 months, discharged within 24 hours of admission, discharged against medical advice, or discharged before medication education was conducted (Figure 1). Patients who met inclusion criteria provided informed consent, received a Mini-Cog screening assessment, and were given the Rapid Estimate of Adult Literacy in Medicine revised (REALM-R) assessment to evaluate health literacy. The REALM-R is a word recognition test designed to identify patients at risk for poor health literacy skills. Patients with REALM-R scores of 6 or less are considered to have low health literacy. ${ }^{15}$ Patients were randomized to receive either the usual care or pharmacist-directed medication evaluation and management as described in Table 1. Patients included in the study were contacted by phone postdischarge, with 3 attempts on consecutive days. Patients who were readmitted as an inpatient or had an ED visit were not contacted for the study after that point.

Patients enrolled in the control group received the usual standard of care by a clinical pharmacist. This included a medication reconciliation completed from the admitting physician's patient history and physical and medication counseling provided by the physician or nursing staff at discharge. Patients were not interviewed face-to-face on admission and did not receive discharge counseling by a pharmacy team member. Patients were assessed daily by the pharmacist for evaluation of the pharmacotherapy plans and presence of MEs or safety-related concerns. The control group received 1 postdischarge phone call from a pharmacist at day 30 to assess for study endpoints of ADEs, MEs, ED visit, and readmission only. The endpoints of ADEs and MEs were determined by professional judgment by the clinical pharmacist based on an algorithm similar to National Coordinating Council for Medication Error Reporting and Prevention, although a specific tool was not utilized.

The study group received face-to-face medication reconciliation on admission by a pharmacist or a pharmacy student. Prior to discharge, a personalized medication plan was created by the pharmacist and discussed with the physician. Medication discrepancies were addressed prior to the discharge instructions being given and discussed with the patient. Medication counseling was performed at discharge by the pharmacist or pharmacy student. Patients received 3 phone calls at 3,14 , and 30 days postdischarge. The presence of ADEs and MEs were evaluated during each phone call. The patients were asked to confirm their medication regimens including drug, indication, dose, route, and frequency. They were also asked questions regarding possible side effects, new symptoms, and any changes to their current therapy. The calls focused on clarifying the pharmacy discharge plan, resolving any unanswered questions or medication-related issues, identifying and overcoming any barriers to adherence, and assistance with providing patients access to medications by contacting pharmacies and physicians to resolve and troubleshoot further prescription claims and clarifications. Pharmacists performed all postdischarge phone calls. Pharmacy students were able to provide face-to-face medication reconciliation upon admission and 


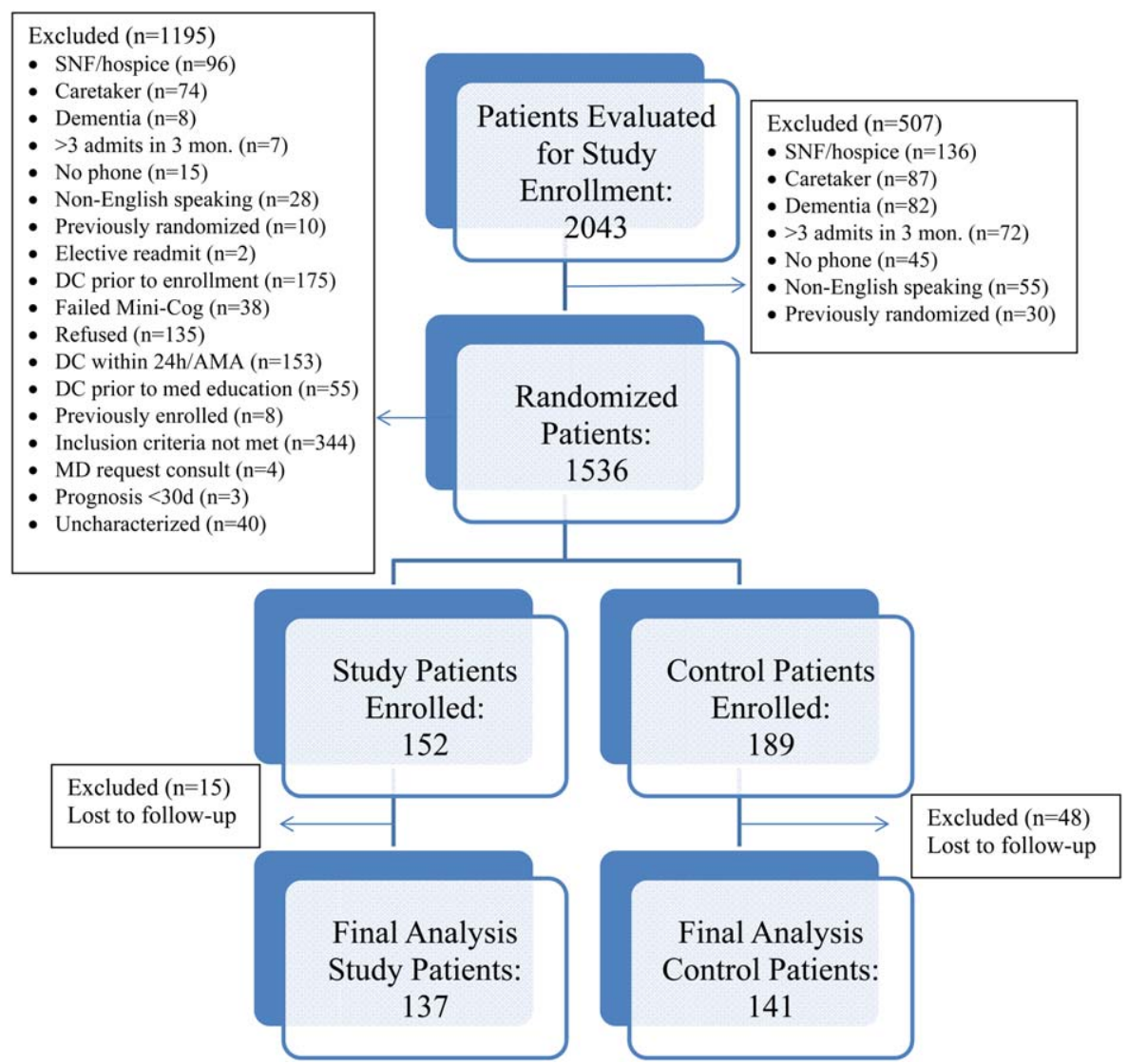

FIG. 1. Enrollment diagram. Abbreviations: AMA, against medical advice; DC, discharge; SNF, skilled nursing facility.

TABLE 1. Comparison of Control Group Versus Study Group Interventions

\begin{tabular}{lll}
\hline Admission Medication Reconciliation & Hospitalist (Confirmation by Pharmacist Reviewing the & Performed by Pharmacy Team Member Face to Face \\
\hline Discharge medication reconciliation & History and Physical Note in Electronic Medical Record) & Pharmacy team member \\
Discharge medication education & Hospitalist & Pharmacy team member \\
Individualized medication plan & Hospitalist and/or nurse & Yes \\
Postdischarge callback day 3 & No & Yes \\
Postdischarge callback day 14 & No & Yes \\
Postdischarge callback day 30 & No & Yes \\
Postdischarge call assessment topic(s) & Yes & ADEs/MEs, ED visits, inpatient readmissions clarify pharmacy/discharge plan, \\
& ADEs/MEs, ED visits, inpatient readmissions & resolve medication-related issues, identify/overcome adherence barriers \\
\hline
\end{tabular}

NOTE: Abbreviations: ADEs, adverse drug events; ED, emergency department; MEs, medication errors.

discharge counseling under the supervision of the pharmacist for the intervention arm.

The patient Hospital Consumer Assessment of Healthcare Providers and Systems (HCAHPS) responses to the medication domain question, "Did you clearly understand the purpose for taking each of your medications at the time of discharge?" were collected for the 2 designated hospitalist units for both the control and study groups. HCAHPS scores were collected at the 6 months point prior to the study initiation and throughout the 6-month study period for the control and intervention groups. A physician and 2 pharmacists, who were blinded to the study ran- domization and results, assessed all Northwestern Memorial Hospital readmissions to determine if the readmissions were medication-related or not.

This study obtained institutional review board approval from Northwestern University.

\section{Data Collected}

Data collected from all patients included demographics (age, sex), payer, reason for admission, number of medications at time of discharge, Charlson Comorbidity Index score, number of high-risk medications prescribed at time of discharge, length of stay, REALM-R score, ADEs, inpatient readmission or ED 
TABLE 2. Patient Demographics

\begin{tabular}{lccc}
\hline & Study, $\mathrm{N}=137$ & Control, $\mathrm{N}=141$ & $P$ Value \\
\hline Sex, male & $52(37.95 \%)$ & $59(41.8 \%)$ & 0.54 \\
Average age, $y$ & 55.4 & 55.8 & 0.87 \\
Average length of stay, d & 5.4 (range, $1-104)$ & 4.6 (range, $0-28)$ & 0.67 \\
Average REALM-R score (range, 0-8) & 6.8 & 6.7 & 0.67 \\
Average total no. of medications & 6.4 & 7.2 & 0.04 \\
Average no. of scheduled medications & 5.7 & 6.2 & 0.15 \\
Average no. of high-risk & 2.2 & 2.3 & 0.64 \\
$\quad$ category medications & & & \\
Reason for admission & & & \\
Cardiovascular disease & $5(3.4 \%)$ & $15(8.3 \%)$ & 0.035 \\
Pneumonia & $11(7.5 \%)$ & $8(4.4 \%)$ & 0.48 \\
Respiratory & $11(7.5 \%)$ & $9(5 \%)$ & 0.65 \\
Infectious disease & $39(26.5 \%)$ & $53(29.3 \%)$ & 0.13 \\
Gastrointestinal & $25(17 \%)$ & $28(15.5 \%)$ & 0.13 \\
Endocrine & $20(13.6 \%)$ & $34(18.8 \%)$ & 0.76 \\
Genitourinary & $0(0 \%)$ & $0(0 \%)$ & 0.05 \\
Hematological & $19(12.9 \%)$ & $20(11 \%)$ & 1 \\
Injury & $10(6.8 \%)$ & $14(7.7 \%)$ & 1 \\
Neurological & $2(1.4 \%)$ & $0(0 \%)$ & 0.52 \\
Heart failure & $4(2.7 \%)$ & $0(0 \%)$ & 0.24 \\
Myocardial infarction & $0(0 \%)$ & $0(0 \%)$ & 0.58 \\
Mental/substance abuse & $1(0.7 \%)$ & $0(0 \%)$ & 1 \\
\hline & & & \\
\hline NOTE: Abbreviations: REALM-R, Rapid Estimate of Adult Literacy in Medicine revised. & \\
& & & \\
& &
\end{tabular}

visit, and the reason for readmission or ED visit. Only the first occurrence was counted for patients with both an ED visit and an inpatient readmission. It was estimated that a sample size of 150 patients in each group would provide $80 \%$ power to demonstrate a $20 \%$ improvement in ADE rates in the study group. Data were analyzed utilizing Fischer exact, $\chi^{2}$, and Student $t$ tests, and multivariate logistic regression as appropriate. Analyses were performed using SAS version 9.4 (SAS Institute, Inc., Cary, NC).

\section{RESULTS}

Over the course of 7 months, 341 patients were enrolled in the study, 189 in the control arm and 152 in the study arm. Forty-eight patients in the control group and 15 patients in the study group were lost to follow-up. The final analysis included 278 patients, 141 in the control group and 137 in the study group. Patients were eligible for study inclusion if they received at least 1 phone call, which resulted in more patients being lost to follow-up in the control arm due to fewer total phone call attempts. Demographic and disposition data for the control and study groups are shown in Table 2. Baseline characteristics between the 2 groups were similar with the exception of total medications at time of discharge. The control group had more total medications at discharge compared to the study group (7.2 vs $6.4, P=0.04)$. The number of high-risk medications and the number of scheduled medications were similar between both groups. During medication reconciliation, 380 discrepancies $(46.2 \%)$ were found in the study group compared to $205(19.9 \%)$ in the control group $(P<0.0001)$. The
TABLE 3. Outcomes of Study Endpoints

\begin{tabular}{|c|c|c|c|}
\hline & $\begin{array}{l}\text { Study Group, } \\
\qquad n=137\end{array}$ & $\begin{array}{c}\text { Control Group, } \\
n=141\end{array}$ & $\begin{array}{c}P \\
\text { Value }\end{array}$ \\
\hline Composite inpatient readmission and ED visit & $34(24.8 \%)$ & $55(38.7 \%)$ & 0.001 \\
\hline ED visits & $6(4.4 \%)$ & $21(14.8 \%)$ & 0.005 \\
\hline Inpatient readmissions & $28(20.4 \%)$ & $34(23.9 \%)$ & 0.43 \\
\hline Medication-related readmissions & $8(23.5 \%)$ & $13(23.6 \%)$ & 1.0 \\
\hline ADEs/MEs reported at 30-day phone call & 11/84 patients & 18/86 patients & 0.22 \\
\hline Days to readmission/ED visit & 7.9 (SD 12.5) & 13.2 (SD 9.61) & 0.03 \\
\hline $\begin{array}{l}\text { Preintervention: HCAHPS scores pertaining } \\
\text { to knowledge of indication of medication } \\
\text { question preintervention }\end{array}$ & \multicolumn{3}{|c|}{$47 \%$} \\
\hline $\begin{array}{l}\text { Postintervention: HCAHPS scores pertaining } \\
\text { to knowledge of indication of medication } \\
\text { question postintervention }\end{array}$ & \multicolumn{3}{|c|}{$56 \%$} \\
\hline
\end{tabular}

NOTE: Abbreviations: ADEs, adverse drug events; ED, emergency department; HCAHPS, Hospital Consumer Assessment of Healthcare Providers and Systems; MEs, medication errors; SD, standard deviation.

higher number of identified discrepancies in the study group was expected due to the fact that the pharmacist did not complete a face-to-face medication history in the control patients. The average length of stay, REALM-R scores, and reason for admissions were similar between the 2 groups (Table 2).

A total of 55 patients $(39 \%)$ in the control arm were readmitted to an inpatient hospital or had an ED visit within 30-days postdischarge compared to 34 patients $(24.8 \%)$ in the study group $(P=0.001)$ (Table 3). Of the patients readmitted to the ED, 21 were enrolled in the control arm $(14.8 \%)$ compared to only 6 patients in the study arm $(4.4 \%)$ $(P=0.005)$. Reviewers concluded that $24 \%$ of the control group readmissions were medication-related versus $23 \%$ of the study group $(P=1.0)$. In total, 78 out of 89 readmissions were to Northwestern Memorial Hospital. Medication-related causes to outside institutions were not evaluated. The causes for all readmissions were not evaluated.

A sensitivity analysis was undertaken to understand the impact of the lost to follow-up rate in both the control and study groups. Undertaking an assumption that all 15 patients lost to follow-up in the study group were readmitted and that 15 of 48 patients lost to follow-up in the control group were readmitted, the intervention continued to show a significant benefit in reduction of composite ED and inpatient readmissions $(35.7 \%$ study group vs $49.6 \%$ control group, $P=0.022$ )

Multivariate logistic regression analysis that controlled for Charlson Comorbidity Index score, length of stay, total number of medications on discharge, and payer type showed an adjusted odds ratio of 0.55 (95\% confidence interval [CI]: $0.32-0.94)$ in the intervention cohort compared to controls for the combined endpoint of readmission and ED visit within 30-days postdischarge. The adjusted odds ratio for 30-day readmission alone was $0.88 \quad(95 \%$ CI: 0.49-1.61). 
Eighteen of the 86 control patients who received a 30-day postdischarge phone call experienced an ADE or ME compared to 11 of the 83 study patients $(P=0.22)$. Patient satisfaction scores of both designated units as represented by the HCAHPS score in the medication knowledge domain increased from the prestudy period. Patients selected "agree" or "strongly agree" only $47 \%$ of the time at the 6-month prestudy point compared to $56 \%$ of the time during the 6 month study period.

\section{DISCUSSION}

Although previous studies show conflicting results regarding the impact of pharmacist interventions on readmissions, our study demonstrated a decrease in the composite measure of inpatient readmissions and ED visits. Its success stresses the need for a comprehensive approach that contains continuity of care by healthcare providers to reconcile and manage medications throughout the hospital stay, extending up to a full month postdischarge with multiple phone calls. This included (1) face-to-face medication reconciliation on admission, (2) development of a personalized medication plan discussed with the patient's physician, (3) addressing any medication discrepancies to the discharge instructions being given to the patient, (4) medication counseling performed at discharge, and (5) 3 postdischarge phone calls at 3,14, and 30 days.

A study conducted in 2001 analyzed the Medicare Current Beneficiary Survey (MCBS) and found that living alone, having limited education, and lack of self-management skills have significant associations with early readmission. ${ }^{16}$ Approximately 80 million Americans have limited health literacy and are associated with poor health outcomes and healthcare utilization as seen in a review completed by Berkman and colleagues. ${ }^{17}$ Because no difference was found between both groups, it would suggest health literacy did not influence or bias the study group. Additionally, no statistically different medication issues, such as total number of medications or rates of ADEs and MEs, were identified in the patients of this study. This may be explained by the small, final population size at the 30-day period or that the impact of the pharmacist intervention did not reach the threshold that this study was powered to detect. Also, a lack of statistical significance may be due to the subjective nature of ADEs/MEs and the prevention of ADEs/ MEs throughout all patients' hospitalizations from the clinical pharmacist's involvement in care, which was not collected. Although a combined endpoint collecting readmission to either the ED or rehospitalization was lower in the intervention cohort, the isolated rehospitalization endpoint was not significantly different between the 2 groups. ED utilization was markedly decreased, but we may have lacked the power to show a statistically significant decrease in rehospitali- zation. These results mirror those of the Project RED (Re-Engineered Discharge) intervention. ${ }^{17}$

HCAHPS surveys are sent to only a small percent of randomly selected patients who are discharged from the hospital. Thus, respondents may or may not have been included in the study, indicating a possible greater impact of the intervention on individual patients than collected. Importantly, the described interventions appeared to improve patients' perception of understanding the purpose of their medications. We found that HCAHPS scores across the 2 units improved, though the intervention only impacted $16.8 \%$ of all patients discharged from these units due to the nature of the survey distribution.

The pharmacists' abilities to educate all eligible patients prior to discharge from 7:30 AM to 4:00 PM each day of the week was a limitation of this study, as some patients were discharged outside of the duty hours. This may have allowed for a differential exclusion and could have led to selection bias. Another limitation is that a large number of patients were lost to follow-up in the control group, likely because the first postdischarge contact with patients was not until the day 30 phone calls. The extensive exclusion criteria caused many patients not to be enrolled. Though the intervention arm received postdischarge phone calls at days 3 and 14, only postdischarge call-backs at day 30 of the intervention arm were compared to the control arm, which could have led to bias in the 30-day analysis of the intervention arm, as patients may have not reported previous issues that were resolved in earlier phone calls. Medication-related readmissions were not statistically different between the groups, which could suggest that the difference in readmissions were not solely due to the intervention, and a decrease in healthcare utilization may be due to chance. The subjective nature of how ADEs and MEs were collected also serves as a limitation, as they were only screened for presence or absence and not classified by severity or category. This study was at a single-center academic institution, which may limit the ability to apply the results to other institutions. Last, outcome assessments relied on participant report, including $\mathrm{ADE}$ and $\mathrm{ME}$ occurrence and presentation at outside hospitals. Future study evaluation conducted as a multicenter design while continuing to strengthen the continuity of the healthcare provider and patient relationship at each intervention would be ideal. Also, having an objective measure of ADEs and MEs with severity categorization would be beneficial.

Compared to previous literature, our study design was unique in the number of phone calls made to patients postdischarge and its prospective, randomized design. In the previously mentioned study by Walker et al., phone calls were made only at days 3 and $30 .{ }^{13}$ Although the majority of readmissions occurred within the first 14 days of discharge, additional visits 
to the ED and readmissions may have been avoided by contacting patients twice within the critical 14-day period. Another distinction of this study design was the expansion of a rather limited and peripheral pharmacist role in transitions of care to a much more integrated participation. We believe the relationship developed between patients and their pharmacy care team provided coordination and the continuity of communication regarding their care. Additionally, our study was unique through the use of pharmacy extenders via fourth-year pharmacy students who were completing their advanced pharmacy practice rotations. Pharmacy extenders can also be certified and trained pharmacy technicians, which many hospitals utilize to perform medication reconciliations at a lower cost than pharmacists. As hospitals face increased demands to shrink budgets due to decreasing reimbursements, healthcare systems will be forced to find creative new ways to use existing resources.

In conclusion, transition of care is a high-risk situation for many patients. A comprehensive approach by healthcare providers, including pharmacists and pharmacy extenders, may have a positive impact in reducing or preventing $\mathrm{ADEs} / \mathrm{MEs}$, inpatient admissions, and ED visits. Although our study focused directly on the impact of a pharmacy care team on transitions-of-care, we cannot conclude this applies strictly to pharmacists. Across the nation, the role of various disciplines of healthcare providers in admission, hospitalization, discharge, and postdischarge is not standardized and varies significantly by institution. Importantly, no mechanism currently exists to directly reimburse for such efforts, but demonstration of cost effectiveness through reduced posthospital utilization may justify this investment for accountable care organizations. ${ }^{18}$

\section{References}

1. Gerhardt G, Yemane A, Hickman P, Oelschlaeger A, Rollis E, Brennan N. Medicare readmission rates show meaningful decline in 2012. Medicare Medicaid Res Rev. 2013;3(2):E1-E11.

2. Feigenbaum P, Neuwirth E, Trowbridge L, et al. Factors contributing to all-cause 30-day readmissions: a structured case series across 18 hospitals. Med Care. 2012:50(7):599-605.

3. Schipper JL, Kirwin JL, Cotugno MC, et al. Role of pharmacist counseling in preventing adverse events after hospitalization. Arch Intern Med. 2006;66:565-571.

4. X - Li J, Young R, Williams MV. Optimizing transitions of care to reduce rehospitalizations. Cleve Clin J Med. 2014;81(5):1-9.

5. Forster AJ, Murff HJ, Peterson JF, Gandhi TK, Bates DW. The incidence and severity of adverse events affecting patients following discharge from the hospital. Ann Intern Med. 2003;138:161-167.

6. Forster AJ, Murff HJ. Adverse drug events occurring following hospital discharge. J Gen Intern Med. 2005;20:317-323.

7. Kerzman H. What do discharged patients know about their medications? Patient Educ Couns. 2005;56:276-282.

8. Dudas V, Bookwalter T, Kerr KM, Pantilat SZ. The impact of telephone calls to patients after hospitalization. Dis Mon. 2002;48:239-248.

9. Kripalani S, Roumie CL, Dalal AK, et al. Effect of a pharmacist intervention on clinically important medication errors after hospital discharge: a randomized trial. Ann Intern Med. 2012;157:1-10.

10. Jack BW, Chetty VK, Anthony D, et al. A reengineered hospital discharge program to decrease rehospitalization: a randomized trial. Ann Intern Med. 2009;150(3):178-187.

11. Al-Rashed SA, Wright DJ, Roebuck N, Sunter W, Chrystyn H. The value of inpatient pharmaceutical counselling to elderly patients prior to discharge. Br J Clin Pharmacol. 2002;54:657-664.

12. Kilcup M, Schultz D, Carlson J, Wilson B. Postdischarge pharmacist medication reconciliation: impact on readmission rates and financial savings. J Am Pharm Assoc (2003). 2013;53(1):78-84.

13. Walker PC, Bernstein SJ, Tucker Jones JN, et al. Impact of pharmacist-facilitated hospital discharge program. Arch Intern Med. 2009;169:2003-2010.

14. Holland R, Desborough J, Goodyer L, et al. Does pharmacist-led medication review help to reduce hospital admissions and deaths in older people? A systematic review and meta-analysis. Br J Clin Pharmacol. 2008;65(3):303-316.

15. Baker D. The meaning and the measure of health literacy. J Gen Intern Med. 2006;21(8):878-883.

16. Arbaje AI, Wolff JL, Yu Q, Powe NR, Anderson GF, Boult C. Postdischarge environmental and socioeconomic factors and the likelihood of early hospital readmission among community-dwelling Medicare beneficiaries. Gerontologist. 2008;48(4):495-504.

17. Berkman ND, Sheridan SL, Donahue KE, Halpern DJ, Crotty K. Low health literacy and health outcomes: an updated systematic review. Ann Intern Med. 2011;155(2):97-107.

18. Fisher ES, McClellan MB, Bertko J, et al. Fostering accountable health care: moving forward in Medicare. Health Affairs. 2009;28(2):219-231. 